\title{
The breakup of intravascular microbubbles and its impact on the endothelium
}

\author{
Wolfgang Wiedemair ${ }^{1,3}$ - Zeljko Tukovic ${ }^{2}$ - Hrvoje Jasak ${ }^{2}$. \\ Dimos Poulikakos $^{1}$ • Vartan Kurtcuoglu ${ }^{3,4} 4_{\mathbb{C}}$
}

Received: 28 November 2015 / Accepted: 27 September 2016

(C) Springer-Verlag Berlin Heidelberg 2016

\begin{abstract}
Encapsulated microbubbles (MBs) serve as endovascular agents in a wide range of medical ultrasound applications. The oscillatory response of these agents to ultrasonic excitation is determined by MB size, gas content, viscoelastic shell properties and geometrical constraints. The viscoelastic parameters of the MB capsule vary during an oscillation cycle and change irreversibly upon shell rupture. The latter results in marked stress changes on the endothelium of capillary blood vessels due to altered MB dynamics. Mechanical effects on microvessels are crucial for safety and efficacy in applications such as focused ultrasound-mediated blood-brain barrier (BBB) opening. Since direct in vivo quantification of vascular stresses is currently not achievable, computational modelling has established itself as an alternative. We have developed a novel computational framework combining fluid-structure coupling and interface tracking to model the nonlinear dynamics of an encapsulated MB in constrained environments. This framework is used to investigate the mechanical stresses at the endothelium resulting from MB shell rupture in three microvessel setups of increasing levels of geometric detail. All configurations predict substantial elevation of up to $150 \%$ for peak wall shear stress upon
\end{abstract}

Vartan Kurtcuoglu

vartan.kurtcuoglu@uzh.ch

1 Laboratory of Thermodynamics in Emerging Technologies, Department of Mechanical and Process Engineering, ETH Zurich, Sonneggstrasse 3, 8092 Zurich, Switzerland

2 Faculty of Mechanical Engineering and Naval Architecture, University of Zagreb, Ivana Lucica 5, 10000 Zagreb, Croatia

3 The Interface Group, Institute of Physiology, University of Zurich, Winterthurerstrasse 190, 8057 Zurich, Switzerland

4 Zurich Center for Integrative Human Physiology, and Neuroscience Center Zurich, University of Zurich, Zurich, Switzerland
MB breakup, whereas global peak transmural pressure levels remain unaltered. The presence of red blood cells causes confinement of pressure and shear gradients to the proximity of the MB, and the introduction of endothelial texture creates local modulations of shear stress levels. With regard to safety assessments, the mechanical impact of MB breakup is shown to be more important than taking into account individual red blood cells and endothelial texture. The latter two may prove to be relevant to the actual, complex process of BBB opening induced by MB oscillations.

Keywords Microbubbles · Shell breakup · Microvessel · Wall shear stress $\cdot$ Geometrical complexity

\section{Introduction}

Microbubbles (MBs) with a viscoelastic encapsulation layer are used as systemic contrast-enhancing agents in medical ultrasound (US) radiology (Quaia 2007). They act as scattering centres for impinging ultrasound waves due to the difference in acoustic impedance between gas content and blood. These ultrasound contrast agents (UCAs) exhibit echogenic properties according to their size, which is typically in the range of $1-10 \mu \mathrm{m}$, gas content and shell properties (Stride and Saffari 2003a). More recently, the same UCAs have been employed in combination with focused ultrasound for the systemic transport and targeted delivery of medication to specific tissues or locations in the body (Ferrara et al. 2007; Hernot and Klibanov 2008). Insonated UCAs are also used to trigger an enhancement of the local permeability of the blood-brain barrier (BBB) (Abbott et al. 2010) for drug transfer (Abbott and Romero 1996; Hynynen 2008; Vykhodtseva et al. 2008) and are employed in a range of other therapeutic applications (Liu et al. 2006; Unger et al. 2004). 
The oscillatory dynamics of the endovascular MB depend on the excitation pressure amplitude, the shell characteristics, liquid properties and the geometrical setting. Typical resonance frequencies are in the $\mathrm{MHz}$ range. Oscillating MBs produce microscopic pulsatile currents in the surrounding liquid. This microstreaming projects oscillatory forces onto nearby boundaries and can cause or augment mechanical or biological effects. The transient local opening of the $\mathrm{BBB}$ is associated with such processes (McDannold et al. 2006), which need to be well controllable in order to prevent irreversible damage to the BBB (Hynynen et al. 2005). The intensity of mechanical surface effects depends on the microstreaming characteristics which are a function of the vibrations of the MB, which in turn can be tuned by US settings and pre-determined by tailoring the shell parameters (Stride 2005).

The encapsulation serves to stabilize the MBs against dissolution and coalescence. It is composed of a thin layer of viscoelastic material that deforms with the expansion and contraction of the $\mathrm{MB}$, rendering the shell characteristics a function of current bubble size. Upon MB compression, the shell is compacted, which can cause a wrinkling of that layer. Such buckling is observed in lipid monolayers (Versluis 2010) and attributed to the congestion of the constant number of lipid molecules in a shell of decreasing size. The same phenomenon is observed when the MB shrinks due to gradual gas elusion (Borden and Longo 2002). The shell loses most of its tension in the buckling state (Marmottant et al. 2005), causing a marked change in the dynamics of the MB, which is otherwise typically dominated by surface tension. During bubble expansion in pressure rarefaction cycles, the viscoelastic shell experiences strain and the tension may surpass a critical limit above which the shell ruptures. The breakup of the encapsulation (Stride and Saffari 2003b) exposes parts of the gas interface directly to the surrounding liquid, while remnants of the shell may still float at the interface. This causes an abrupt change in MB characteristics, leading to a change in oscillation patterns and subsequent dissolution of the MB (de Jong et al. 2009).

The rupture of MBs inside vessels of the circulatory system changes the dynamics of the involved MB. The altered microstreaming can result in a marked change in mechanical stresses at the endothelium. The generated shear may trigger biological responses, such as an increase in BBB permeability (McDannold et al. 2006), or may damage cell membranes ( $\mathrm{Wu} 2002$ ). Hence, the stress on the endothelial cell membranes exerted by MB breakup is of interest for the assessment of potential tissue response or damage and ultimately for a risk-benefit assessment.

Breakup of different types of encapsulated MBs has been experimentally observed and characterized by Moran et al. (2000), and the influence of ultrasound parameters on the MB oscillations was assessed using acoustic and optical feedback (Dayton et al. 1999; de Jong et al. 2009). Direct optical observation employing ultrahigh-speed cameras (Chin et al. 2003) allowed to follow the transient kinetics of the MB (de Jong et al. 2000), while the mechanical damage caused by MB oscillations and breakup in vessels was studied in gel phantoms (Caskey et al. 2009). Direct observation of $\mathrm{MB}$ oscillation in microvessels was reported by Caskey et al. (2007), and the resulting microvessel deformation in ex vivo vessels was characterized by Chen et al. (2011). However, experimental assessment is currently limited to kinetic observations and a posteriori damage assessment since the small time and length scales of the involved processes pose enormous challenges to in situ measurement of dynamics quantities like shear and pressure at the vessel walls.

The dynamics of a single MB with clean surface in an infinite fluid space exposed to an ultrasound field is accurately described by the Rayleigh-Plesset equation (RPE) (Plesset and Prosperetti 1977). An analytical model including the effect of a solid shell was proposed by Church (1995) and adapted for thin encapsulating layers by Hoff et al. (2000). Based on experimental findings, Sarkar et al. (2005) proposed a model for MBs with infinitely thin viscoelastic shells characterized by size-dependent properties. Taking into account shell buckling, a finite elastic range of the encapsulation and shell rupture, Marmottant et al. (2005) developed an extension towards a three-state model categorized by the tension of the interface. Further refined rheological shell models incorporating non linear shell viscosity were proposed by Li et al. (2013) and Doinikov et al. (2009). The modelling of MBs inside rigid tubes is achieved by introducing an effective mass term (Klotz and Hynynen 2010). However, most analytical models are restricted to a spherically oscillating MB in simple geometrical settings.

Numerical modelling permits greater flexibility in studying complex, coupled setups that are more relevant to practical, medical purposes. The oscillation of MBs in flexible, plain tubes (Ye and Bull 2006) was studied, and lumped parameter solid models allowed investigation of the interaction of MBs with compliant microvessels (MVs) of variable size (Qin and Ferrara 2006). The mechanical stresses at the endothelial interface were assessed using coupled solid and fluid models (Hosseinkhah et al. 2013; Wiedemair et al. 2012). The breakup of MBs near rigid walls (Hsiao and Chahine 2013) and inside MVs (Hosseinkhah et al. 2015) was also studied numerically.

Currently available numerical models often simplify MVs as liquid-filled straight tubes with plain walls. This geometric simplification neglects many features of the microvasculature, such as the presence of red blood cells (RBCs) in close proximity to the $\mathrm{MB}$ and the texture of the MV resulting from the endothelial cell morphology. RBCs may influence the local microflow and thereby have an impact on mechanical stress at the endothelium. A more detailed representation 
of the endothelial wall morphology could cause alterations of wall shear patterns, since this mechanical parameter is very sensitive to the local surface configuration. Therefore, neglecting a subset of the geometric features may unduly reduce model fidelity and ultimately limit the level of validity of the assessed mechanical wall parameters such as transmural pressure and wall shear stress.

The purpose of this study is to investigate the effect of including additional geometric MV features that can be of importance, such as RBCs and endothelial texturing. While the representations of the employed geometrical structures are still idealized, they allow for general conclusions about the value of increasing geometric complexity. Mechanical parameters derived from models of enhanced complexity may prove relevant to safety assessment of medical treatments involving MBs. Moreover, a detailed and more realistic representation of the involved mechanical force patterns may contribute to elucidating the underlying mechanisms of BBB opening, a process that is currently not fully understood (Sheikov et al. 2004).

To enable this investigation, we introduce a novel fully generic numerical framework to treat the complex coupling of viscoelastic MB dynamics, blood microflow and vessel structural dynamics in the event of $\mathrm{MB}$ shell breakup. It combines the ability to accurately model non-spherical oscillations of MBs with size-dependent viscoelastic shell properties and the coupling to arbitrarily shaped solid domains. This versatile tool allows the study of changes in the wall parameter patterns for increasingly detailed setups.

\section{Methods}

We introduce a computational framework to model the changes in mechanical stresses on the endothelium caused by the breakup of a single encapsulated microbubble inside a MV at increasing levels of geometric detail. The interaction of multiple, physically distinct dynamic domains is implemented by means of iterative, bidirectional coupling. The coupling of intraluminal microflow and structural mechanics of the vessel is achieved by employing a fluid-structure interaction (FSI) algorithm. We translate the three-state approach by Marmottant et al. (2005) into an adaptive fluid boundary condition for an interface tracking (IFT) algorithm, which allows embedment of the MB dynamics into the model. This computational framework permits the modelling of compliant MVs hosting an encapsulated MB with variable viscoelastic properties immersed in blood plasma and surrounded by RBCs (Fig. 1). The RBC spacing of 5-8 $\mu \mathrm{m}$ was calculated based on the average concentration of RBCs in human blood (McHedlishvili and Varazashvili 1980) and the mean capillary diameter (Cassot et al. 2006). This setup is exposed to oscillating pressure fields of varying amplitude,

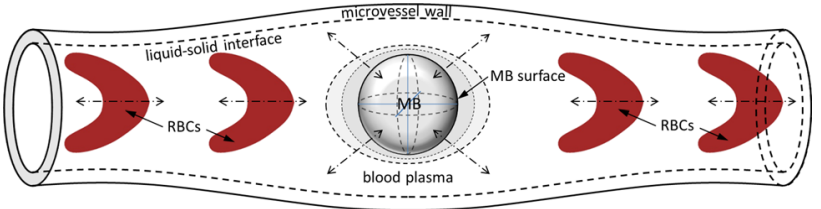

Fig. 1 The geometrical setup comprises a single microbubble embedded in a train of RBCs inside a compliant MV. The interfaces between $\mathrm{MB}$ and blood plasma as well as the liquid-solid interface between blood plasma and the MV wall are deformable. The RBCs are motile in axial direction

while the bubble dynamics, microstreaming and endothelial interface stresses are monitored.

Blood plasma is modelled as an incompressible, Newtonian fluid, and the structural component of the MV as well as the RBCs is regarded as homogeneous, isotropic, linear elastic solid (Tukovic and Jasak 2007). These domains are coupled using an FSI algorithm (Kuttler et al. 2010) with Arbitrary Lagrangian-Eulerian (ALE) formulation of the fluid constitutive equations on a dynamically adapting, unstructured computational mesh (Jasak and Tukovic 2007). An interface quasi-Newton technique with inverse Jacobian from a least-squares model (IQN-ILS) (Degroote et al. 2009) is employed to optimize stability and convergence of the computation. Both computational domains are discretized using a second-order accurate cell-centred unstructured finite volume scheme. The algebraic systems are solved iteratively, using a segregated solution procedure based on the PISO algorithm (Issa 1986) for the pressure-velocity coupling in the fluid and a segregated algorithm for determining the three components of the displacement increment in the linear momentum conservation law written in updated Lagrangian formulation for the solid (Tukovic and Jasak 2007). The transient problem is treated in a time-marching manner with uniform time step size and an implicit, second-order accurate time integration scheme (Ferziger and Peric 1995). Wiedemair et al. (2012) provides details on the employed coupling algorithm and a thorough description of the solid and fluid modelling, including the formulations of constitutive equations as well as references to model derivation.

The dynamics of the MB are coupled with the fluid domain via an adaptable, deformable boundary at the MB-liquid interface. The basic algorithm for tracking the MB interface is described by Tukovic and Jasak (2012) and was employed for modelling the interaction of multiple MBs by Wiedemair et al. (2014). This IFT algorithm is extended and generalized by the introduction of viscoelastic shell properties that scale with the dynamic size of the MB during oscillation.

\subsection{Analytical MB model}

A MB with a thin viscoelastic encapsulation can be characterized by its equilibrium radius $a_{0}$ as well as the dynamic viscosity $\mu_{s}$ and the tension $\sigma_{s}$ of its shell (Chatterjee 
and Sarkar 2003). When the MB radius changes during US-induced oscillation, the viscoelastic properties of the MB shell may be altered (Tu et al. 2009; van der Meer et al. 2007) due to the compaction or rarefaction of its constituent molecules. Marmottant et al. (2005) suggest that $\mu_{s}$ scales with the inverse of the current bubble radius $a$ according to

$\mu_{s}=\frac{\kappa_{s}}{a}$

with shell dilatational viscosity $\kappa_{s}$.

The surface tension of the MB can be written in a piecewise form as

$\sigma(A)= \begin{cases}0 & \text { if } A \leq \tilde{A} \\ \sigma_{S}(A) & \text { if } \tilde{A}<A<\hat{A} \\ \sigma_{0} & \text { if } A \geq \hat{A}\end{cases}$

where $\tilde{A}$ is the surface area below which buckling occurs and $\hat{A}$ denotes the critical maximum surface area at which the shell ruptures. Experimental findings (Borden and Longo 2002; Versluis 2010) indicate that the shell loses tension in the buckling state. In the elastic regime, $\sigma_{s}$ is proportional to the surface area $A$ of the MB according to

$\sigma_{s}=\chi\left(\frac{A}{\tilde{A}}-1\right)$

where $\chi$ is the elastic shell compression modulus. $\kappa_{s}$ and $\chi$ are constants determined by the composition of the shell. The elastic regime is limited by the buckling behaviour towards small surface areas and the shell rupture towards large surface areas.

Shell rupture occurs when surpassing the critical maximum surface area $\hat{A}$. After rupture of the shell, the gas content is directly exposed to the surrounding liquid, resulting in a constant surface tension $\sigma_{0}$ for the gas-liquid interface (Paul et al. 2010). The breakup surface area

$\hat{A}=\tilde{A}\left(1+\frac{\sigma_{c}}{\chi}\right)$

is determined from the maximum tension $\sigma_{c}$ the shell can sustain. $\sigma_{c}$ is usually of the same order of magnitude as $\sigma_{0}$ (Stride and Saffari 2003b) with proposed values for the upper end of the elastic regime ranging from $\sigma_{c}=\sigma_{0}$ (Versluis 2010) to several times $\sigma_{0}$ (Marmottant et al. 2005).

Rupture changes the shell characteristics permanently to $\sigma=\sigma_{0}$ and $\mu_{s}$ reduces to the residual viscosity $\mu_{r}$. That quantity is a fraction of the original $\mu_{s}$ and accounts for the effect of the shell remnants, of which large portions will still reside at the interface. For spherical MBs, the equivalent buckling radius $\tilde{a}$ and the equivalent breakup radius $\hat{a}$ can be employed as more intuitive limits of the linear regime.
The response of a MB with the described shell characteristics and polytropic gas content to a transient pressure variation $P_{a}(\mathrm{t})$ can be described by the modified RPE

$$
\begin{aligned}
\rho_{l}\left(a \ddot{a}+\frac{3}{2} \dot{a}^{2}\right)= & \left(P_{0}+\frac{2 \sigma\left(A_{0}\right)}{a_{0}}\right)\left(\frac{a_{0}}{a}\right)^{3 \gamma} \\
& -P_{0}-\frac{2 \sigma(A)}{a}-4 \frac{\dot{a} \mu_{t}}{a}-P_{a}(t),
\end{aligned}
$$

where dots denote derivatives with respect to time, $\rho_{l}$ is the liquid density, $\gamma$ is the polytropic exponent, $P_{0}$ denotes equilibrium pressure and $\mu_{t}$ is the total viscosity defined as

$\mu_{t}=\mu_{l}+\mu_{s}$,

where $\mu_{l}$ is the dynamic viscosity of the liquid.

\subsection{Numerical MB model}

A more general case of non-spherical MB oscillation can be modelled by tracking the MB-liquid interface in a 3D numerical simulation employing the finite volume formulation. We consider a deformable liquid domain, representing the blood compartment of the setup, where the flow of incompressible fluid is governed by mass and momentum conservation according to the Navier-Stokes equations written in ALE formulation. A portion of the boundary of this domain coincides with the interface between blood and MB. At this interface, an adaptable boundary condition that is updated according to the dynamic conditions in the MB is applied. This MBliquid interface $\Gamma_{b}$ is iteratively deformed until normal stress equilibrium

$$
\left.P\right|_{\Gamma_{b}}=P_{g}-\kappa \sigma(A)-\left.2 \mu_{t}(\kappa) \nabla_{s} \cdot \mathbf{v}\right|_{\Gamma_{b}}
$$

is reached. Here, $P$ is the liquid pressure, $\kappa$ denotes twice the local surface curvature calculated by $\kappa=-\nabla_{s} \cdot \tilde{\mathbf{n}}$, where $\nabla_{s}$ is the surface gradient and $\tilde{\mathbf{n}}$ is the surface normal unit vector, $\mathbf{v}$ represents fluid velocity, and $P_{g}$ is the transient internal gas pressure of the MB. $P_{g}$, considered homogenous throughout the bubble, is derived from the polytropic law

$P_{g}=P_{g, o}\left(\frac{V_{b, o}}{V_{b}}\right)^{\gamma}$,

where $P_{g, 0}$ is the equilibrium gas pressure, $V_{b, 0}$ is the MB equilibrium volume, and $V_{b}$ is the current $\mathrm{MB}$ volume.

The solution procedure relies on an interface tracking approach (Tukovic and Jasak 2012) to determine the exact position of the MB interface assuming zero mass transfer across $\Gamma_{b}$. The sharp delineation of the MB interface is essential for accurate evaluation of Eq. (7), which is dominated by the surface tension term due to large $\kappa$ values as a result of 
the small size of the MB. We developed a numerical solver tailored to address the combination of FSI at the endothelial interface with IFT at the MB surface in the numerical platform OpenFOAM (Jasak et al. 2007; Weller et al. 1998). This solver relies on the iterative solution of the solid-fluid coupling problem using a segregated FSI algorithm with nested iteration loops addressing the IFT of the MB surface inside the fluid domain.

With the application of transient pressure boundary conditions at the vessel inlets and at the outer MV surface, the MB immersed in blood plasma at the centre of the vessel is subjected to a transient excitation signal. $P_{a}$ is assumed spatially homogenous throughout the considered domain because the configuration is much smaller than the US wavelength. Since the typical blood flow velocity of $1 \mathrm{~mm} \mathrm{~s}^{-1}$ in capillaries (Fischer et al. 1996; Stucker et al. 2004) is about three orders of magnitude smaller than the radial MB interface velocity, the MB will not be displaced significantly from its initial location during the small time window relevant to MB oscillation and breakup, which is in the range of $\mu$ s. Therefore, advection due to physiologic blood flow is neglected.

The deformation of the MB according to the surrounding flow conditions is mediated by Eq. (7) and will in turn induce oscillatory flow to the surrounding liquid. The impact of the generated microstreaming on the displacement of the luminal vessel interface $\Gamma_{s f}$ can be determined through FSI. The forces acting on this interface are evaluated in terms of wall shear stress

$\boldsymbol{\tau}_{w s s}=\left.\mu_{l} \tilde{\mathbf{n}} \cdot \nabla(\mathbf{v}-(\mathbf{v} \cdot \tilde{\mathbf{n}}) \tilde{\mathbf{n}})\right|_{\Gamma_{s f}}$

and transmural pressure

$P_{\mathrm{tm}}=\left.P\right|_{\Gamma_{s f}}-\left(P_{i}+P_{a}\right)$,

where $P_{i}$ represents the static pressure in the interstitial space outside the vessel and $\Gamma_{s f}$ indicates that the quantities are evaluated at the luminal vessel interface. These two quantities, termed mechanical wall parameters, characterize the normal and transverse stresses on the MV endothelium. The stresses at the endothelial vessel interface before and after the breakup of the shell of an encapsulated MB with sizedependent shell characteristics are investigated. They are relevant to cavitation-induced damage and are hypothesized to play a key role in BBB opening (Chen and Konofagou 2014; McDannold et al. 2008; Nyborg 2001; Wamel et al. 2006).

All studied setups comprise a single MB of equilibrium radius $a_{0}=1 \mu \mathrm{m}$ with $\tilde{a}=0.985 \mu \mathrm{m}$ and shell properties $\kappa_{s}=15 \mathrm{nN} \mathrm{s} \mathrm{m}^{-1}, \chi=1.0 \mathrm{~N} \mathrm{~m}^{-1}$ and $\mu_{r}=0.4 \mu_{s}$ with a polytropic exponent of $\gamma=1.095$, suitable for sulphur hexafluoride gas as employed in SonoVue ${ }^{\circledR}$ UCAs (Marmottant et al. 2005). A breakup tension of $\sigma_{c}=0.115 \mathrm{~N} \mathrm{~m}^{-1}$ is assumed, corresponding to $\hat{A}=13.6 \mu \mathrm{m}^{2}$ or $\hat{a}=1.04 \mu \mathrm{m}$ for an equivalent spherical shape. The MB is located at the centre of a $l_{t}=40 \mu \mathrm{m}$ long, compliant MV with luminal radius $r_{t}=3 \mu \mathrm{m}$ (Cassot et al. 2006) and $0.5 \mu \mathrm{m}$ average wall thickness (Bertossi et al. 1997), mimicking a portion of a capillary blood vessel. The vessel wall has a mass density $\rho_{v}$ of $1200 \mathrm{~kg} \mathrm{~m}^{-3}$, and Young's modulus $E_{v}$ is set to $8 \mathrm{MPa}$ (Miao et al. 2008). The liquid properties are adjusted to represent blood plasma with $\rho_{l}=1030 \mathrm{~kg} \mathrm{~m}^{-3}$ (Lowe 1987) and $\mu_{l}=1.5 \times 10^{-3} \mathrm{~kg} \mathrm{~m}^{-1} \mathrm{~s}^{-1}$ (Haidekker et al. 2002).

A configuration often employed for the modelling of oscillating endovascular MBs is a single MB placed in the centre of a blood plasma containing straight tube with plain luminal interface. We use this as the basis of our study, referring to it as Setup I. The inherent rotational and central symmetries are exploited to accelerate computation, and the configuration is resolved with a hexahedral, block-structured computational mesh. We evolve this into Setup II by adding pairs of RBCs upstream and downstream of the MB according to Fig. 1 and investigate these cells' impact on the wall parameters. While passing through a MV, the tips of the deformed, parachute-shaped RBCs (Secomb et al. 2006) slide along the endothelium (Pries et al. 2000). In the chosen axisymmetric configuration, the RBCs can move in axial direction driven by the oscillation of the MB. To represent the textured MV surface, endothelial cells lining the MV are approximated by hexagonal honeycomb structures arranged in nominal blood flow direction in Setup III. They feature a bulge at the centre representing the protruding cell nucleus region and indentations at the cell junction. The geometries in Setups II \& III are resolved using a polyhedral, unstructured computational mesh. Average mesh densities in the three setups are 281219 computational cells per $\mu \mathrm{m}^{3}$, corresponding to average resolutions of $0.15-0.17 \mu \mathrm{m}$, with interfaces being resolved much finer than bulk regions.

The configurations are exposed to a sinusoidal pressure field $P_{a}(\mathrm{t})$ with transiently growing pressure amplitude $P_{\mathrm{ex}}$ at an excitation frequency of $f_{\mathrm{ex}}=2.6 \mathrm{MHz}$ (Marmottant et al. 2005; Schlachetzki et al. 2002). MBs with thin viscoelastic shells can typically sustain acoustic pressures in the range of $100-150 \mathrm{kPa}$ (de Jong et al. 2009), depending on $f_{\text {ex }}$ and the exact shell configuration. $P_{\mathrm{ex}}$ is linearly ramped up from an initial setting $P_{\mathrm{ex}, i}=130 \mathrm{kPa}$ to a final value $P_{\mathrm{ex}, f}=$ $180 \mathrm{kPa}$ during a ramp time interval $\Delta t_{r}=5 \mu \mathrm{s}$ and stays constant at $P_{\mathrm{ex}, f}$ for $t>\Delta t_{r}$. The parameters are chosen such that the MB initially oscillates in the stable regime and then gradually approaches and surpasses the breakup threshold.

The employed computational framework is based on a combination of two algorithms, FSI and IFT with constant parameters. Validation studies for both methods have been performed and are provided in Wiedemair et al. (2012) and Wiedemair et al. (2014), respectively. A validation of the novel generalized IFT algorithm for scalable viscoelastic 
shell properties is provided in the "Appendix" section, where we established close correspondence of numerically determined results with analytical predictions from Eq. (5) and also find good agreement with a reference case.

\section{Results}

We shall increase the complexity of the geometric setup (Fig. 1) in three stages by introducing additional features. The impact of those model adjustments on $\tau_{\mathrm{wss}}, P_{\mathrm{tm}}$ and their derivatives, collectively referred to as wall parameters, is monitored. Starting from a straight tube with plain walls of fixed thickness (Setup I), we shall assess the effect of introducing RBCs into the MV (Setup II) and then of adding a texture that mimics the structure of endothelial cells at the vascular interface (Setup III).

\subsection{Setup I: Plain vessel without RBCs}

Figure 2 shows an arrangement of plots for the time window around the shell rupture in a straight tube with texture-free walls (Setup I). The plots include the excitation pressure signal $P_{a}(t)$, the average MB radius $\bar{a}$, the maximum of the magnitude of $\boldsymbol{\tau}_{\mathrm{wss}}\left(\left|\boldsymbol{\tau}_{\mathrm{wss}}\right|_{\max }\right)$ and the maximum transmural pressure, $P_{\mathrm{tm} \text {,max }}$. To discard inflow and boundary effects at the vessel ends, a sampling domain spanning $15 \mu \mathrm{m}$ to both sides of the location of the MB rather than the entire tube is considered for determining the maxima. A linear increase of excitation pressure amplitude is imposed over the first $5 \mu \mathrm{s}$.

The MB shell breaks at a local minimum of $P_{a}$ at $t_{b}=$ $4.32 \mu \mathrm{s}$ (dashed line in Fig. 2), leading to an immediate sharp increase in $\bar{a}$. This growth of vibration amplitude is accompanied by an increase of $\left|\tau_{\mathrm{wss}}\right|_{\max }$ to approximately 2.5 times its pre-breakup value. $P_{\mathrm{tm}, \max }$ shows a change in its transient pattern after shell rupture, but no appreciable alteration in peak values. The occasionally observable abrupt changes of slope in the patterns of $P_{\mathrm{tm} \text {,max }}$ and $\left|\boldsymbol{\tau}_{\mathrm{wss}}\right|_{\max }$ are the result of a switch of spatial location of the maximum value.

We observe a distinct preference for compression as evident in the temporal pattern of the bubble radius curve, which reaches values far below $a_{0}$. This kinematic comportment was also pointed out by Marmottant et al. (2005) and experimentally confirmed (de Jong et al. 2007; Versluis 2010). The change of slope in the compression phase is caused by a decrease in $\sigma(\mathrm{A})$ and a simultaneous increase of $\mu_{s}(\kappa)$. It marks the transition of the MB dynamics from the surfacetension-dominated regime to buckling comportment.

To analyse the spatial stress distribution, we sampled wall shear stress in axial direction, $\tau_{\mathrm{wss}, \mathrm{z}}$, and transmural pressure along a line, $\Xi$, parallel to the longitudinal vessel axis $z$ on the luminal interface. Since the setup is axially symmetric, this sampling represents the stress patterns on the entire

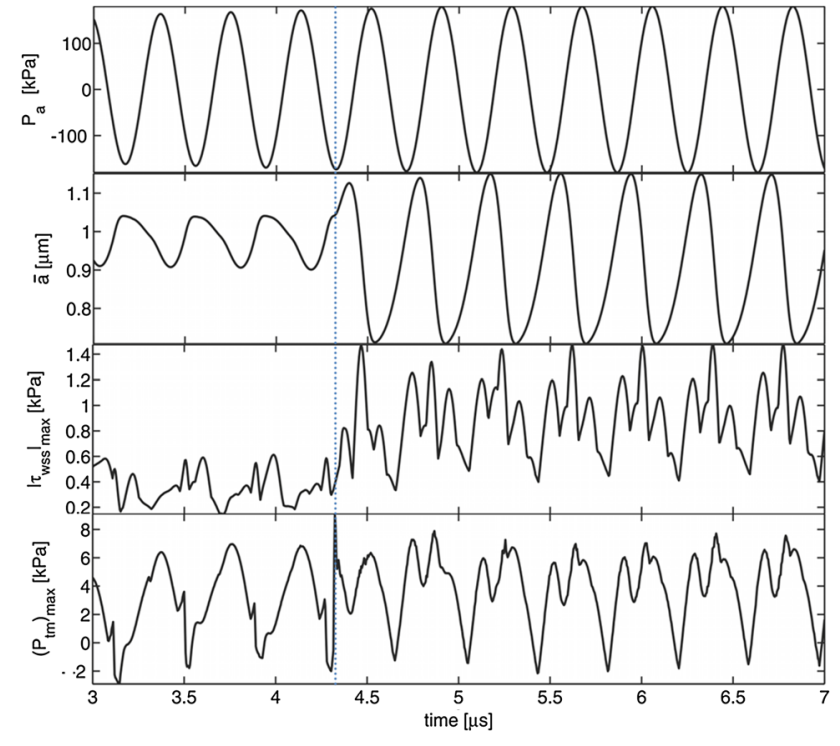

Fig. 2 Temporal evolution around the time point of shell rupture (dotted line) of $P_{a}, \bar{a},\left|\tau_{\mathrm{wss}}\right|_{\max }$ and $P_{\mathrm{tm}, \max }$ patterns for $P_{\mathrm{ex}, i}=130 \mathrm{kPa}$, $P_{e x, f}=180 \mathrm{kPa}$ and $\Delta t_{r}=5 \mu \mathrm{s}$ in a vessel with plain luminal walls and without RBCs (Setup I). As $P_{a}$ increases linearly from $P_{\mathrm{ex}, i}$ to $P_{\mathrm{ex}, f}$, shell rupture takes place at $t_{b}=4.32 \mu \mathrm{s}$, causing a marked change in MB oscillation pattern and amplitude, and a substantial increase in $\left|\tau_{\mathrm{wss}}\right|_{\max }$. The $P_{\mathrm{tm} \text {,max }}$ pattern changes shape, but peak values do not deviate significantly from pre-rupture levels

luminal surface. We stack the data sampled along $\Xi$ at consecutive time steps to allow for a spatio-temporal analysis of the wall parameters and their axial gradients (Fig. 3 ). $\tau_{\mathrm{wss}, \mathrm{Z}}$ and $P_{\mathrm{tm}}$ show oscillatory temporal patterns that change after MB shell breakup. The initially weak transient variation of $P_{\mathrm{tm}}$ in the region around the location of the MB $(z=0)$ increases in amplitude after $t_{b}$, and its shape changes from a single pressure peak to that of a pressure peak pair during each excitation pressure oscillation cycle. This can also be seen in the $P_{\mathrm{tm}, \max }$ pattern in Fig. 2. At the same time, the pressure oscillation in distal regions continues with prerupture amplitude.

$\tau_{\mathrm{wss}, \mathrm{z}}$ increases substantially along $\Xi$ following shell rupture, and isolated local extrema become more apparent. Pronounced peaks of $\tau_{\mathrm{wss}, \mathrm{Z}}$ establish immediately lateral to the flow stagnation point located at $z=0$. The gradients of $\tau_{\mathrm{wss}, \mathrm{z}}$ and $P_{\mathrm{tm}}$ along $z$ show wave-like patterns and increase markedly after $t_{b}$.

\subsection{Setup II: Plain vessel containing RBCs}

The assessment of $P_{a}(t), \bar{a},\left|\tau_{\mathrm{wss}}\right|_{\max }$ and $P_{\mathrm{tm}, \max }$ for a texture-free vessel containing RBCs, performed in the same fashion as for Setup I, reveals that the MB comportment does not change considerably in the presence of RBCs, with MB shell breakup again taking place at $t_{b}=4.32 \mu \mathrm{s}$ (Fig. 4). While the peak values of the wall parameters stay in the 

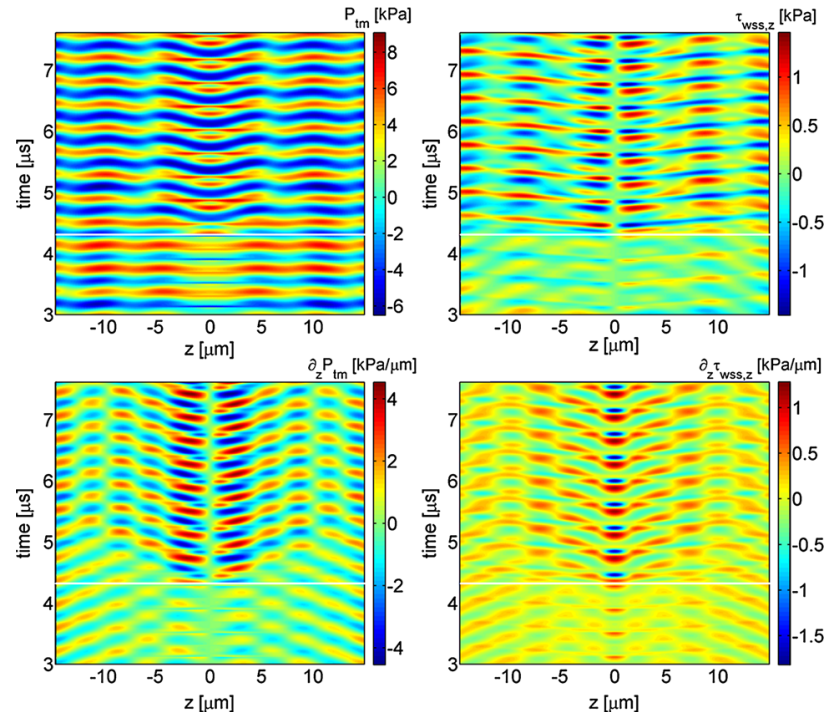

Fig. 3 Spatio-temporal behaviour of wall parameters along the axial sampling line $\Xi$ for Setup I. $P_{\mathrm{tm}}$ shows a layered temporal comportment with ripples in $z$ direction and a marked change in pattern at the MB location $(z=0)$ after shell rupture at $t_{b}=4.32 \mu \mathrm{s}$ (white horizontal line). The peak $P_{\mathrm{tm}}$ values do not change appreciably due to the rupture. $\tau_{\mathrm{wss}, \mathrm{z}}$ increases substantially after shell rupture, and isolated local extrema become more apparent. The axial gradients of both parameters increase after $t_{b}$ and exhibit a wave-like pattern

same range as without RBCs, their transient patterns have noticeably different shapes.

A study of the spatio-temporal comportment of $P_{\mathrm{tm}}$ and $\tau_{\text {wss, }}$ (Fig. 5) along $\Xi$ reveals the impact of RBCs on the wall parameters. The $P_{\mathrm{tm}}$ pattern is generally similar to Setup $\mathrm{I}$, but shows clear damping in the $\mathrm{RBC}$ regions (marked by dashed lines). The peak pressure amplitude in the vicinity of the $\mathrm{MB}$ is greatly enhanced after shell rupture, but surpasses pre-rupture $P_{\mathrm{tm}}$ levels only marginally. Moreover, a transient pressure peak pair establishes in this region after $t_{b}$. The major variations in axial gradients are contained between the innermost RBCs. Substantially lower gradients are observed in the space between the, with respect to the MB, proximal and distal RBCs.

$\tau_{\mathrm{wss}, \mathrm{Z}}$ increases substantially after $t_{b}$ to similar values as in Setup I, but exhibits a different, more layered pattern with jumps in the downstream $(z>0)$ RBC locations and local maxima in their upstream positions. This loss of symmetry of the upstream and downstream regions, as compared to Setup I, is caused by the parachute shape of the RBCs. Only the symmetry about the longitudinal axis is still preserved in Setup II. Similar to the $P_{\mathrm{tm}}$ gradients, large values of the axial $\tau_{\mathrm{wss}, \mathrm{z}}$ gradients are contained between the innermost RBCs, while lower gradients are observed in the inter-RBC space between the two upstream and the two downstream RBCs.

Overall, the introduction of RBCs causes a confinement of the major stress variations in the vicinity of the $\mathrm{MB}$

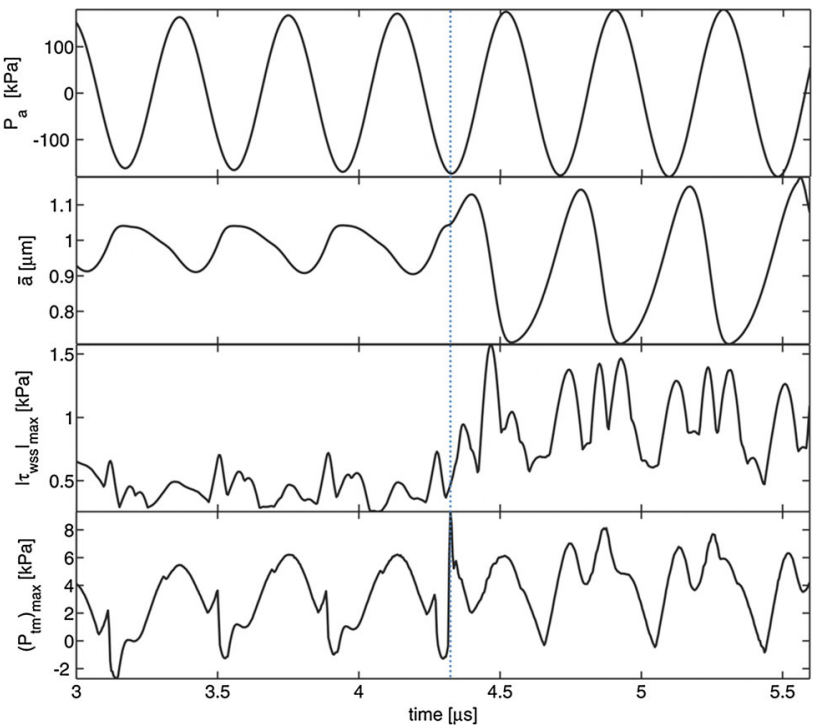

Fig. 4 Temporal evolution around the time of shell rupture (dotted line) of $P_{a}, \bar{a},\left|\tau_{\mathrm{wss}}\right|_{\max }$ and $P_{\mathrm{tm}, \max }$ with $P_{\mathrm{ex}, i}=130 \mathrm{kPa}, P_{\mathrm{ex}, f}=180$ $\mathrm{kPa}$ and $\Delta t_{r}=5 \mu \mathrm{s}$ in a vessel with plain luminal walls containing RBCs (Setup II). The peak values of the wall parameter are similar to Setup I, but the transient comportment shows noticeable differences in the observed fluctuation patterns

and creates a distinction between patterns in upstream and downstream regions, which is more pronounced in the $\tau_{\mathrm{wss}, \mathrm{Z}}$ patterns and its respective gradients than in the $P_{\mathrm{tm}}$ distributions.

\subsection{Setup III: Texturized vessel containing RBCs}

Figure 6 shows temporal patterns of $P_{a}, \bar{a},\left|\tau_{\mathrm{wss}}\right|_{\max }$ and $P_{\mathrm{tm}, \max }$ for a textured MV containing RBCs. The MB shell ruptures at $t_{b}=4.7 \mu \mathrm{s}$, which is later than in the previous configurations. The peak values of $P_{\mathrm{tm}}$ and $\left|\tau_{\mathrm{wss}}\right|$ are elevated by $20 \%$ and $30 \%$, respectively, but the qualitative transient comportment of the wall parameters is similar to the previous setups with substantial increase in $\left|\tau_{\mathrm{wss}}\right|_{\max }$ after shell rupture and a change in the pattern of $P_{\mathrm{tm}, \max }$ without an increase in overall peak values.

The spatio-temporal comportment of $P_{\mathrm{tm}}$ and $\tau_{\mathrm{wss}, \mathrm{z}}$ along $\Xi$, which was placed such that it does not run along a cell-cell junction or directly across a cell nucleus bulge, is displayed in Fig. 7. Qualitatively, little difference to the patterns found for Setup II is visible, except for a damping of $\tau_{\mathrm{wss}, \mathrm{z}}$ at the location where $\Xi$ crosses a cell junction (black arrow). The increase in peak values at a location where $\Xi$ passes next to a cell nucleus bulge (white arrow) is not discernible due to overlay with already present regions of high $\tau_{\text {wss, } z}$. However, it can be assessed from slightly higher peak values in the $\tau_{\mathrm{wss}, \mathrm{z}}$ islands immediately left of the MB location as compared to their right counterparts. 

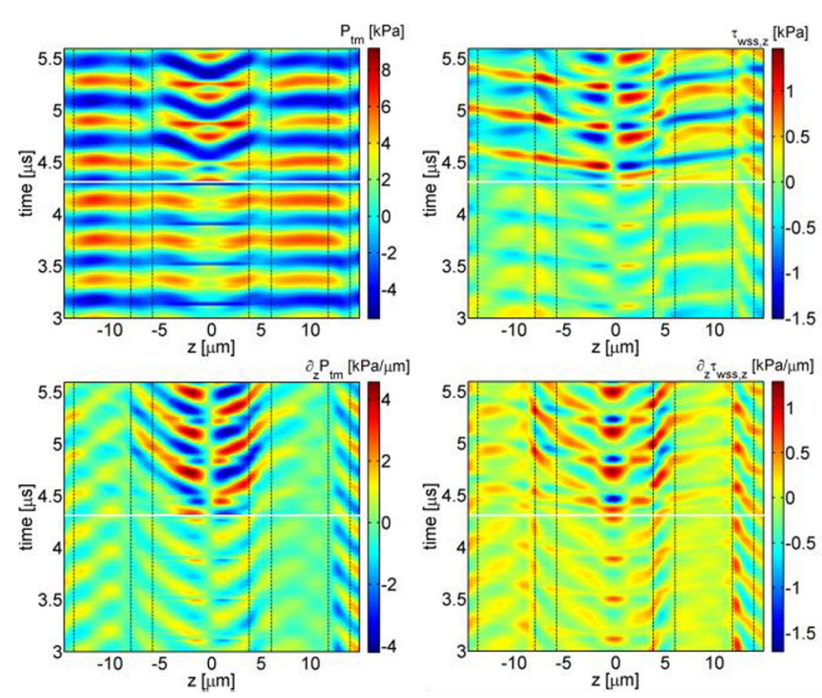

Fig. 5 Spatio-temporal behaviour of wall parameters along $\Xi$ for Setup II. $P_{\mathrm{tm}}$ shows a layered temporal comportment with noticeable damping in areas where the distance between vessel wall and RBC is below $1 \mu \mathrm{m}$ (narrow regions between vertical dashed lines) and a marked change in pattern at the MB location $(z=0)$ after rupture. The peak values of $P_{\mathrm{tm}}$ do not change appreciably due to shell rupture, but its axial gradients increase after $t_{b}$. Areas of high spatial transmural pressure gradient are visible between the innermost RBCs, followed by low gradient regions in the gaps between the RBCs. $\tau_{\mathrm{wss}, \mathrm{z}}$ rises strongly after shell rupture along with its axial gradients, where again confinement effects due to RBCs are observed

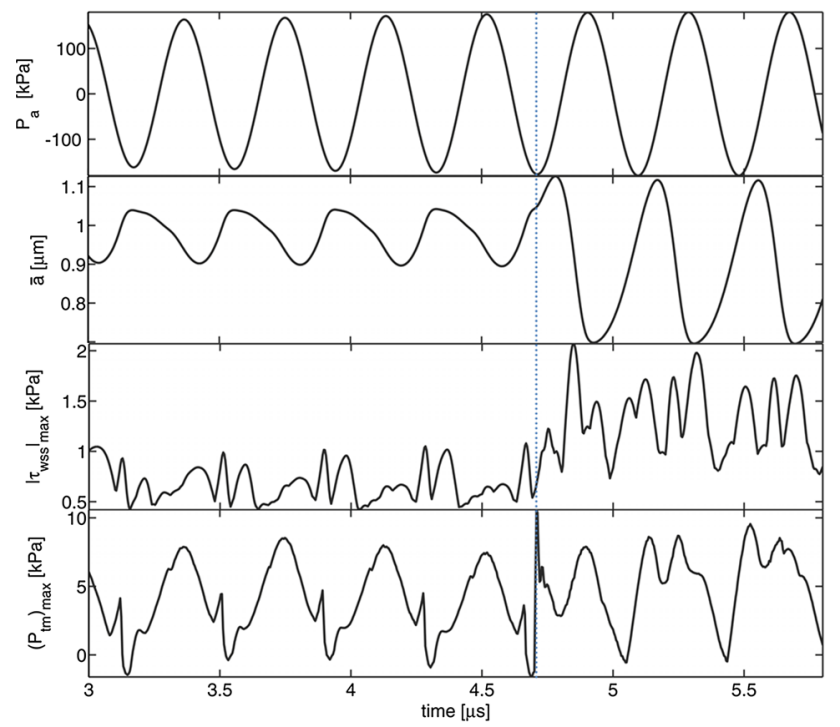

Fig. 6 Temporal evolution around the time point of shell rupture (dotted line) of $P_{a}, \bar{a},\left|\tau_{\mathrm{wss}}\right|_{\max }$ and $P_{\mathrm{tm} \text { max }}$ for Setup III. Shell breakup occurs later than in previous configurations, and the peak values of the wall parameters are elevated by $20-30 \%$. The transient patterns of $\left|\boldsymbol{\tau}_{\mathrm{wss}}\right|_{\text {max }}$ and $P_{\mathrm{tm} \text {,max }}$ are similar to those seen in Setup II

Axial gradients along a longitudinal sample line cannot be considered fully representative for this setup because the surface texture breaks rotational symmetry. Significant changes in wall parameters will appear in directions other than the ves-
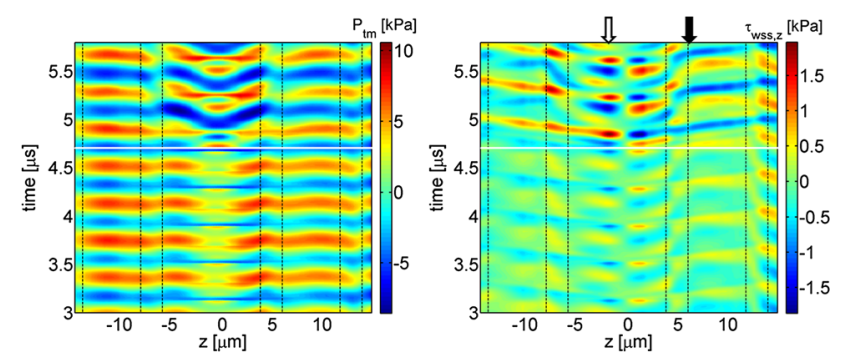

Fig. 7 Spatio-temporal behaviour of wall parameters along $\Xi$ for a configuration with RBCs and vessel wall texture. No major differences in patterns compared to Setup II can be observed. A discernible reduction of $\tau_{\mathrm{wss}, \mathrm{z}}$ is seen around a cell junction (black arrow), while an increase at the site of a cell nucleus bulge (white arrow) is barely visible due to overlap with an also otherwise present region of strong shear

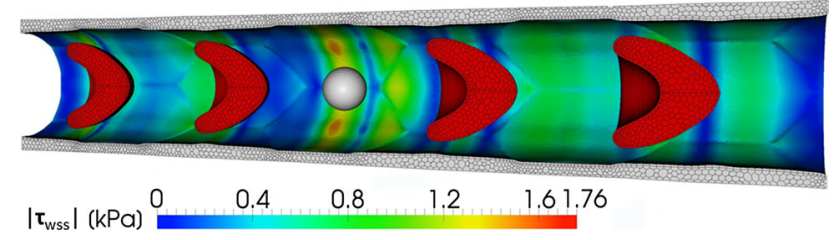

Fig. 8 Cutaway of a textured vessel containing RBCs (Setup III) with the luminal vessel face coloured to represent $\left|\boldsymbol{\tau}_{\mathrm{wss}}\right|$. The largest shear values are contained between the innermost RBCs. Maxima are visible at the central cell nucleus bulges, and cell-cell interfaces have slightly lower $\left|\tau_{\mathrm{wss}}\right|$ values

sel axis at cell nucleus bulges and cell-cell junctions, where the latter could be of particular relevance with respect to possible endothelial damage or changes in permeability. The shear stress magnitude distribution on the vessel interface at $t=5.6 \mu \mathrm{s}$ is shown in a cutaway in Fig. 8. Shear stress levels increase around the protruding cell cores and are diminished at cell-cell junctions. This distribution gives rise to strong, highly localized gradients.

Overall, the introduction of MV wall morphology damps the amplitude of MB oscillation, delaying MB shell breakup. While $\boldsymbol{\tau}_{\mathrm{wss}}$ and $P_{\mathrm{tm}}$ patterns do not differ appreciably from Setup II, the peak values increase due to variations in local MV cross section. These peak values are observed highly localized at the protruding bulges and receding cell-cell junctions of the endothelial cell pattern.

\section{Discussion}

We have analysed a series of three increasingly detailed setups for the modelling of ultrasound-induced MB vibrations and shell breakup inside a MV to assess the influence of geometric simplifications on the prediction of wall parameters. System dynamics were calculated using a novel computational framework featuring interface tracking capability combined with fluid-structure interaction. We assessed the spatial and temporal comportment of wall parameters 
for all setups and identified newly emerging features when increasing the level of geometric detail. The assessed wall parameters have an impact on the MV endothelium, and their magnitude and spatial distribution are relevant with respect to inducing cell damage (Wu 2002) or evoking biological responses (McDannold et al. 2006).

The rupture of the MB shell was achieved by exposing the setup to transient pressure variations of increasing amplitude until the surface stresses surpassed a critical $\sigma_{c}$. The presence of RBCs did not noticeably change the dynamics of the MB (Stride and Saffari 2004) and the time $t_{b}$ until shell breakup was unaltered compared to Setup I. However, shell breakup was delayed in Setup III, where the cross section of the vessel lumen is locally narrowed by portions of the endothelial cells protruding from the wall. This imposes an increased damping effect on the forced MB oscillations due to confinement (Klotz and Hynynen 2010; Wiedemair et al. 2014). Consequently, the MB oscillates with reduced amplitude and surpasses the rupture threshold at a later time and at higher $P_{\mathrm{ex}}$.

A substantial increase of peak $\boldsymbol{\tau}_{\mathrm{wss}}$ values after shell rupture was observed in all three investigated setups (Figs. 2, 4, 6). The wall shear scales with the surface normal gradient of the flow velocity (9) of the microstreaming caused by the MB vibrations. This velocity is considerably increased after shell breakup due to the rise in MB oscillation amplitude observed in all setups. This translates to a considerable increase in liquid volume displacement. Hence, elevated post-rupture levels of $\boldsymbol{\tau}_{\mathrm{wss}}$ are a direct consequence of the increased MB oscillation amplitude after shell breakup and should therefore be expected in all setups.

Unaltered overall peak levels of $P_{\mathrm{tm}}$ before and after shell rupture were found for all investigated setups (Figs. 2, 4, 6). However, the local pressure levels in the vicinity of the MB rise, and a double-peak morphology establishes in the postrupture patterns. The pressure levels at the MB interface are determined according to Eq. (7) by shell tension $\sigma$, surface curvature $\kappa$, viscous forces and internal gas pressure, which is a function of MB volume according to Eq. (8). The change of tension from $\sigma_{s}$ to $\sigma_{0}$ and the reduction of $\mu_{s}$ to $\mu_{r}$ due to shell breakup are compensated by an increase in surface velocity and a larger variation in MB volume and, consequently, in internal gas pressure. This equilibration effect can leave the liquid pressure levels at $\Gamma_{b}$ and hence $P_{\mathrm{tm}}$ basically unaltered.

Overall, shell breakup in MVs is characterized by an immediate substantial increase in oscillation amplitude and wall shear stress accompanied by no appreciable change in transmural pressure. This qualitative comportment is observed irrespective of the level of geometric complexity. It implies that any consideration on mechanical MV loading by encapsulated MBs should include assessment of the dynamics of the ruptured bubble state, because this generally creates the highest $\tau_{\mathrm{wss}}$.
The introduction of RBCs (Setup II) leads to a change in shape of the spatio-temporal $\tau_{\mathrm{wss}, \mathrm{z}}$ patterns. The axial displacement of the RBCs with the flow causes the fluid in the upstream and downstream inter-RBC gaps to move like a plug. This creates homogenous $\tau_{\mathrm{wss}, \mathrm{z}}$ strips in these regions (Fig. 5), as compared to the observed island structures in Setup I (Fig. 3). In contrast, spatially confined and locally varying $\boldsymbol{\tau}_{\mathrm{wss}, \mathrm{z}}$ patterns are observed in proximity to the MB. Taken together, these patterns cause strong local gradients, which are confined to the vicinity of the MB, and low gradient values beyond the proximal RBCs. Therefore, the endothelium directly adjacent to the MB location experiences maximum levels of strain, while more distal regions are less exposed.

The spatio-temporal patterns of $P_{\mathrm{tm}}$ are less affected by the presence of RBCs than those of $\tau_{\mathrm{wss}}$. Because of the plug flow in the inter-RBC space, homogeneous pressure layers are observed. These layers are disrupted by faster flow through the narrow annular passage between RBC tips and vessel interface, causing locally reduced pressure levels. Due to this layering and the increased pressure levels close to the MB, the high-intensity regions of the $P_{\mathrm{tm}}$ gradients are confined between the innermost RBCs. Low levels are visible beyond the proximal RBCs (Fig. 5). Consequently, the maximum levels of transverse strain are imposed on the endothelium directly adjacent to the MB location.

The introduction of RBCs into the MV (Setup II) adds the aspect of confinement of wall parameter variations to the vicinity of the MB. This setup indicates that neighbouring blood cells have substantial impact on local microstreaming and hence on the local MV loading. The fully motile, perfectly parachute-shaped RBCs aligned along the vessel axis used in our model may in fact underestimate confinement effects caused by stacked, tilted and deformed RBCs. Blood cells should thus be taken into consideration when determining the location of maximum vessel straining.

The peak levels of $\boldsymbol{\tau}_{\mathrm{wss}}$ at feature locations of the endothelial wall texture (Setup III) are substantially higher than for texture-free setups (Setups I and II). $\boldsymbol{\tau}_{\mathrm{wss}}$ is increased at cell nucleus bulges and decreased in cell-cell indentations. Both phenomena can be explained by the, respectively, enhanced and reduced exposure of these structures to flow and hence to shearing. The local surface configuration thus significantly modulates $\boldsymbol{\tau}_{\mathrm{wss}}$. The surface features introduced in Setup III are rather shallow and could be much more pronounced in real MVs. Hence, the impact of the real endothelial morphology can be expected to exceed the levels encountered in this study.

The introduction of endothelial cell texturing results in a substantial increase in overall $P_{\mathrm{tm}}$ levels. However, $P_{\mathrm{tm}}$, unlike $\tau_{\mathrm{wss}}$, does not depend directly on the local surface shape. Therefore, we cannot infer that more pronounced texturing will necessarily lead to significantly higher $P_{\mathrm{tm}}$. In this 
context, the overall reduction in vessel lumen cross section, which may result from more prominent endothelial texturing, is relevant.

Vessel wall texture causes a substantial increase in peak wall parameters and creates localized $\boldsymbol{\tau}_{\mathrm{wss}}$ peaks at feature locations of the endothelial cells, causing punctual loading of the MV. This implies that the strain on the MV wall is not only confined to the close proximity of the $\mathrm{MB}$, but is also focused on some critical subcellular features.

The magnitude of $P_{\mathrm{tm}}$ and $\boldsymbol{\tau}_{\mathrm{wss}}$ observed prior to MB shell breakup agrees with values previously derived with different models (Hosseinkhah and Hynynen 2012; Wiedemair et al. 2012). Post-rupture wall parameter levels are in the same range as reported by (Hosseinkhah et al. 2015) using a different numerical approach and a model equivalent in complexity to Setup I. To our knowledge, no experimental data on the dynamic loading of MVs in the presented parameter range are currently available.

Beyond the surface texture introduced in Setup III, the inner surface of a real blood vessel is covered by an endothelial surface layer (ESL). This approximately $0.5 \mu \mathrm{m}$ thick layer consists of macromolecules that are, in part, anchored in the cell membrane (Pries et al. 2000). The ESL could be modelled as a porous medium, partially impeding liquid motion and hence impacting local flow patterns near the endothelium. However, limited availability of mechanical material properties precludes proper modelling of the ESL. Taking the ESL into account would likely result in a reduction of shear stress on the endothelium itself. However, shear stress would be transmitted from the ESL surface by the torque of glycocalyx protein strands to their anchor points at the cell membrane (Pries et al. 2000). The membrane-bound macromolecules of the ESL are known to act as mechanotransducers and play a role in the regulation of cell function (Davies et al. 1997). Therefore, the ESL could act as a stress mediator between the flow and the cell membrane.

Sliding of RBCs past the ESL should reduce flow through the annular gaps around the RBCs substantially, increasing the level of confinement created by the presence of RBCs. Hence, taking the ESL into account would likely further increase the reported compartmentalization of shear gradients and pressure gradients as well as the bulk flow character in the distal intra-RBC gaps. Finally, the ESL reduces the effective luminal cross section of the vessel with respect to liquid motion. Such reduction increases the damping of MB oscillations (Klotz and Hynynen 2010; Wiedemair et al. 2014), which in turn leads to shell breakup at higher $P_{\mathrm{ex}}$.

The introduced geometrical refinements to the model setup discussed in this study are not exhaustive. Parameters like RBC spacing, MV size and shape of the endothelial cells could be varied. Also, non-symmetric setups with offaxis MBs, tilted RBCs or a bent MV could offer additional insight. While the exact distribution of $P_{\mathrm{tm}}$ and $\boldsymbol{\tau}_{\mathrm{wss}}$ would change with altered flow patterns in a modified geometrical setting, the qualitative comportment of these parameters in the event of MB shell rupture is expected to be preserved. The presented computational framework can handle these modified geometries provided the MB remains entirely immersed in plasma. In contrast, the modelling of MBs that are in direct contact with the endothelium or with RBCs would require additional treatment of contact and wetting, which is not implemented in the present framework.

The MB shell fracture process itself was not modelled in this study, since an additional coupling to a two-dimensional dynamic system at a different time scale was beyond the scope of this work. The fracture was assumed to happen instantaneously because the fracture time is much smaller than a MB oscillation cycle. Short-term dynamic phenomena that might cause instantaneous peaks of wall parameters are therefore not covered by this approach. Subsequent to the shell fracture, molecular species such as proteins dissolved in the plasma might adsorb on the liquid-gas interface (Fang and Szleifer 2001), thereby changing its viscoelastic properties. However, the time scale of the diffusion processes required for this (Putnam 1975), and in particular the time required for proteins to reach the interface by passing through the MB's momentum boundary layer, is substantially larger than multiple periods of MB oscillation. Adsorption is therefore not considered for the short time interval investigated in this study. Nonlinear viscous terms were omitted in the shell model because appropriate choice of initial viscoelastic properties allows for a comparable fidelity of the model while maintaining the computational complexity at an acceptable level.

Experimental investigation of the modelled processes would pose great technical challenges due to the small time and length scales involved. It is, indeed, likely beyond the reach of current technology. Nevertheless, the following ideas may prove useful as starting points for experimental examination of at least parts of the presented phenomena: In vitro setups based on cultured endothelial layers mimicking the BBB have been produced for various purposes (Patabendige et al. 2013; Zhang et al. 2006). MBs immersed in liquid could be positioned close to such a cell layer grown on a flat substrate and excited by US. Employing a highspeed camera (Chin et al. 2003) would allow the recording of MB kinematics and a comparison to the computational predictions. However, this approach would not provide values of local shear stress and pressure. It is, furthermore, not clear how the MBs can be steadied close enough to the cell layer throughout the entire process and how the response of the flat in vitro BBB would compared to the real, tubular barrier. Alternatively, a high-frequency oscillator, e.g. piezoelectric actuator, positioned in close proximity to the in vitro cell layer could be employed to generate controlled shear and pressure conditions similar to the ones reported by our model. 
The BBB response could then be assessed by tracking the passage of solutes across the endothelium. Yet it is unclear how exactly the shear and pressure gradients predicted by our model could be achieved, and the precise monitoring of highly localized shear stresses and pressure poses a great challenge.

Although direct experimental validation is currently not possible and despite the discussed limitations in scope, this study demonstrates the effects of geometric simplifications on the computational prediction of microvascular wall stresses during MB oscillation and breakup.

\section{Conclusions}

The most significant change in mechanical wall parameters found in this study is the substantial rise in $\boldsymbol{\tau}_{\mathrm{wss}}$ caused by the rupture of the MB shell. This phenomenon is common to all three investigated setups and is locally modulated by the introduction of RBCs and endothelial cell patterning. With regard to the safety assessment of treatments including MBs, it appears to be sufficient to account for MB shell rupture in the basic geometric setup treated here. The application of conservative safety margins in clinical practice would most likely supersede any local modulations caused by RBCs and cell texturing.

On the other hand, the observed confinement and localization effects may prove to be highly relevant to advancing the knowledge on the mechanisms of BBB opening through MB oscillations. Here, the differences in stress exposure along the endothelial cell membrane as well as the compartmentalization caused by RBCs could be of critical importance. Most prominently, the confinement of stress gradients to the vicinity of the MB and the pronounced local modulation of $\boldsymbol{\tau}_{\mathrm{wss}}$ at the endothelial cell features offer detailed insight into the mechanical vessel loading on a microscopic scale. Correlating the predicted locations of maximum mechanical stresses with sites of increased trans-endothelial substance transport could help to further elucidate the chain of processes involved in BBB opening. A controlled reproduction of numerically attained shear levels, patterns and gradients on endothelial layers in vitro could be used to find such correlation.

Acknowledgments The authors gratefully acknowledge the funding of this research by the Swiss National Science Foundation through NCCR Co-Me and NCCR Kidney.CH.

\section{Appendix: Validation of the IFT algorithm}

An IFT algorithm with a modified pressure boundary condition according to Eq. (7) is employed in this study to model the dynamics of a non-spherically oscillating encapsulated
MB immersed in liquid. It allows for variations of $\sigma_{s}$ and $\mu_{s}$ as a function of MB size, including abrupt changes at shell breakup or during buckling. This algorithm is validated with respect to analytical predictions from Eq. (5).

The validation setup comprises a single MB of equilibrium radius $a_{0}=0.975 \mu \mathrm{m}$ with variable settings for $\kappa_{s}$ and $\chi$. This MB is enclosed in a fluid sphere of radius $R_{l}=100 \mu \mathrm{m}$, mimicking an infinitely large liquid envelope. The liquid is considered to be water with $\rho_{l}=1000 \mathrm{~kg} \mathrm{~m}^{-3}$ and $\mu_{l}=10^{-3} \mathrm{~kg} \mathrm{~m}^{-1} \mathrm{~s}^{-1}$. This setup is exposed to a transient pressure field at various excitation frequencies $f_{\text {ex }}$ and either constant or linearly growing excitation amplitude $P_{\mathrm{ex}}$.

In a first step, the conformity with predictions in the elastic regime and for buckling is established. The maximum tension that the shell can sustain is set to $\sigma_{c}=1 \mathrm{~N} \mathrm{~m}^{-1}$, which is deliberately high to preclude shell rupture at this stage of the validation process. Figure 9 shows a comparison between the transient radius plots acquired with IFT and predicted by the modified RPE in Eq. (5) for the oscillation of a MB driven by an US field of $f_{e x}=2.9 \mathrm{MHz}$ and $P_{\text {ex }}=130 \mathrm{kPa}$. The shell properties were chosen to fit a SonoVue ${ }^{\circledR}$ UCA with $\kappa_{s}=15 \mathrm{nN} \mathrm{s} \mathrm{m}^{-1}, \chi=1 \mathrm{Nm}^{-1}$ and $\tilde{a}=a_{0}=0.975 \mu \mathrm{m}$ (Marmottant et al. 2005). We quantify the conformity between the numerical and the analytical results using the maximum of the transient relative difference

$\varepsilon(t)=\left|\frac{a_{a}(t)-a_{\mathrm{n}}(t)}{a_{a, \max }-a_{a, \min }}\right|$,

where $a_{a}(t)$ represents the transient analytically determined MB radius and $a_{n}(t)$ represents the transient numerically determined MB radius, while $a_{a, \max }$ and $a_{a, \min }$ denote the overall maximum and minimum of $a_{a}(t)$. The analytically predicted and the numerically modelled comportments show excellent agreement with $\varepsilon_{\max }=0.45 \%$ in a simulation

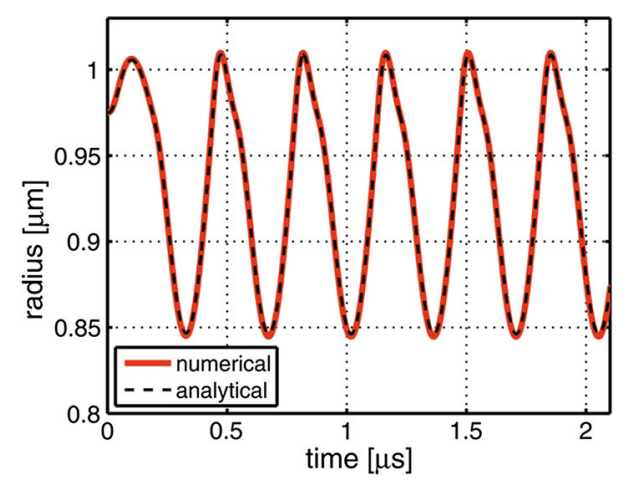

Fig. 9 Comparison of radius response curves from theoretical predictions and numerical results for a configuration with $f_{\mathrm{ex}}=2.9 \mathrm{MHz}$, $P_{\mathrm{ex}}=130 \mathrm{kPa}, \kappa_{s}=15 \mathrm{nN} \mathrm{sm}^{-1}, \chi=1 \mathrm{Nm}^{-1}$ and $\tilde{a}=\mathrm{a}_{0}=$ $0.975 \mu \mathrm{m}$. The numerically determined $a(t)$ shows excellent agreement with the analytical prediction, having a relative deviation of less than $0.45 \%$ 

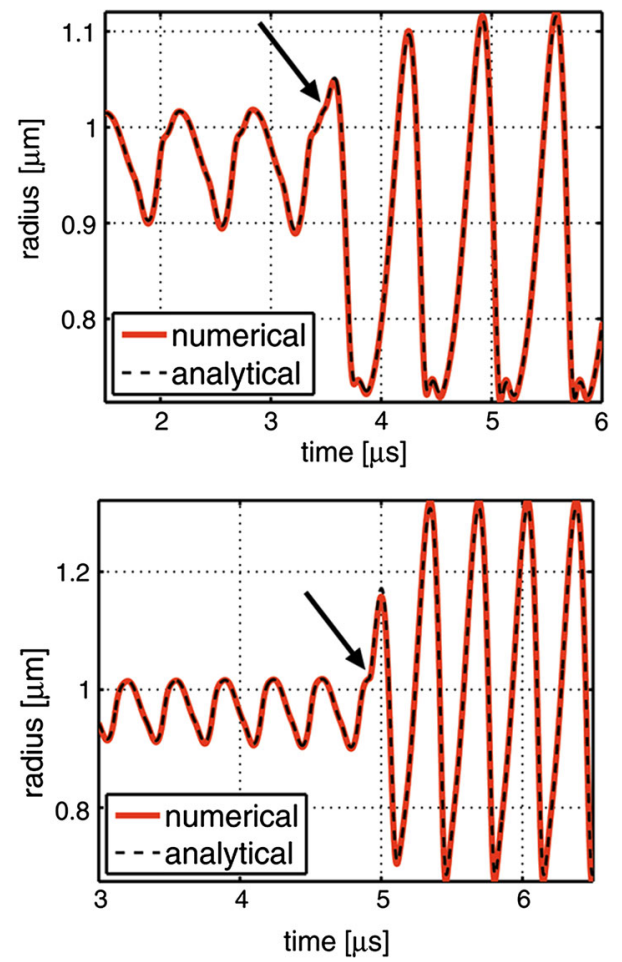

Fig. 10 Comparison between numerical results and analytical prediction for shell break up of two MBs with viscoelastic encapsulation of $a_{0}=0.975 \mu \mathrm{m}$ and $\tilde{a}=0.95 \mu \mathrm{m}$. An encapsulated $\mathrm{MB}$ of $\kappa_{s}=7.2 \mathrm{nN} \mathrm{s} \mathrm{m}^{-1}, \chi=0.55 \mathrm{Nm}^{-1}, \sigma_{c}=0.0615 \mathrm{Nm}^{-1}$ and $\mu_{r}=0.4 \mu_{s}$ (top) exposed to $f_{\mathrm{ex}}=1.5 \mathrm{MHz}, P_{\mathrm{ex}, i}=100 \mathrm{kPa}$ and $P_{\mathrm{ex}, f}=130 \mathrm{kPa}$ with $\Delta t_{r}=5 \mu \mathrm{s}$ shows breakup at $3.48 \mu \mathrm{s}$. Another $\mathrm{MB}$ of $\kappa_{s}=15 \mathrm{nN} \mathrm{sm}^{-1}, \chi=1 \mathrm{~N} \mathrm{~m}^{-1}, \sigma_{c}=0.148 \mathrm{Nm}^{-1}$ and $\mu_{r}=0.4 \mu_{s}$ breaks up at $4.92 \mu \mathrm{s}$ when excited with $f_{\text {ex }}=2.9 \mathrm{MHz}$, $P_{\mathrm{ex}, i}=150 \mathrm{kPa}$ and $P_{\mathrm{ex}, f}=210 \mathrm{kPa}$ with $\Delta t_{r}=5 \mu \mathrm{s}$. Both cases show close agreement between analytical prediction and numerical results

interval of $10 \mu \mathrm{s}$. The numerically acquired data also closely correspond to the results based on simulations using the same parameters published by Marmottant et al. (2005). As expected, the radius pattern exhibits compression preference (de Jong et al. 2007; Versluis 2010) and a change in slope during contraction.

After checking the predictive capability of the numerical model for the elastic regime and the buckling state, we examine its ability to model shell rupture. To achieve a transition from oscillation with encapsulation to oscillation without encapsulation, $P_{\mathrm{ex}}$ is ramped up from an initial $P_{\mathrm{ex}, i}$ to a final value $P_{\mathrm{ex}, f}$ over a time interval $\Delta t_{r}$ and stays constant at $P_{\mathrm{ex}, f}$ for $t>\Delta t_{r}$. Figure 10 displays a portion of the radius-time curves for two configurations with the instants of breakup indicated by arrows. Both encapsulated MBs have a buckling radius of $\tilde{a}=0.95 \mu \mathrm{m}$.

The top panel of Fig. 10 shows results obtained for $f_{\mathrm{ex}}=$ 1.5 MHz, pressure amplitude buildup from $P_{\mathrm{ex}, i}=100 \mathrm{kPa}$ to $P_{\mathrm{ex}, f}=130 \mathrm{kPa}$ over $\Delta t_{r}=5 \mu \mathrm{s}$ and shell properties of $\kappa_{s}=7.2 \mathrm{nN} \mathrm{s} \mathrm{m}^{-1}, \chi=0.55 \mathrm{~N} \mathrm{~m}^{-1}$ (Gorce et al. 2000), $\sigma_{c}=0.0615 \mathrm{~N} \mathrm{~m}^{-1}$ and $\mu_{r}=0.4 \mu_{s}$. The shell's breakup tension is surpassed at $P_{\mathrm{ex}}=121 \mathrm{kPa}$ and $t_{b}=3.48 \mu \mathrm{s}$, after which the oscillation amplitude still keeps growing with increasing $P_{\mathrm{ex}} \cdot \varepsilon_{\max }$ in the elastic regime does not surpass $0.6 \%$ and peaks at $1.9 \%$ after shell breakup.

Figure 10 (bottom) displays findings for the oscillation of a MB with $f_{e x}=2.9 \mathrm{MHz}, P_{\mathrm{ex}, i}=150 \mathrm{kPa}, P_{\mathrm{ex}, f}=$ $210 \mathrm{kPa}, \Delta t_{r}=5 \mu \mathrm{s}, \kappa_{s}=15 \mathrm{nN} \mathrm{s} \mathrm{m}^{-1}, \chi=1 \mathrm{~N} \mathrm{~m}^{-1}$, $\sigma_{c}=0.148 \mathrm{~N} \mathrm{~m}^{-1}$ and $\mu_{r}=0.4 \mu_{s}$. Shell breakup occurs at $4.92 \mu \mathrm{s}$ and $P_{\mathrm{ex}}=209 \mathrm{kPa}$. Subsequently, $a(t)$ quickly stabilizes to a new pattern with constant amplitude. $\varepsilon_{\max }$ in the elastic regime does not surpass $0.4 \%$ and increases to $2.4 \%$ after shell breakup.

All three presented setups show an agreement between numerical results and analytical predictions with a difference below $1 \%$ before shell rupture and lower than $2.5 \%$ after rupture.

\section{References}

Abbott NJ, Romero IA (1996) Transporting therapeutics across the blood-brain barrier. Mol Med Today 2:106-113

Abbott NJ et al (2010) Structure and function of the blood-brain barrier. Neurobiol Dis 37:13-25

Bertossi M et al (1997) Ultrastructural and morphometric investigation of human brain capillaries in normal and peritumoral tissues. Ultrastruct Pathol 21:41-49

Borden MA, Longo ML (2002) Dissolution behavior of lipid monolayer-coated, air-filled microbubbles: effect of lipid hydrophobic chain length. Langmuir 18:9225-9233

Caskey CF et al (2007) Direct observations of ultrasound microbubble contrast agent interaction with the microvessel wall. J Acoust Soc Am 122:1191-1200

Caskey CF et al (2009) Microbubble tunneling in gel phantoms. J Acoust Soc Am 125:EL183-EL189

Cassot $\mathrm{F}$ et al (2006) A novel three-dimensional computer-assisted method for a quantitative study of microvascular networks of the human cerebral cortex. Microcirculation 13:1-18

Chatterjee D, Sarkar K (2003) A Newtonian rheological model for the interface of microbubble contrast agents. Ultrasound Med Biol 29:1749-1757

Chen H, Konofagou EE (2014) The size of blood-brain barrier opening induced by focused ultrasound is dictated by the acoustic pressure. J Cereb Blood Flow Metab 34:1197-1204

Chen $\mathrm{H}$ et al (2011) Blood vessel deformations on microsecond time scales by ultrasonic cavitation. Phys Rev Lett 106:034301

Chin CT et al (2003) Brandaris 128: a digital 25 million frames per second camera with 128 highly sensitive frames. Rev Sci Instrum 74:5026-5034

Church CC (1995) The effects of an elastic solid-surface layer on the radial pulsations of gas-bubbles. J Acoust Soc Am 97:1510-1521

Davies PF et al (1997) Spatial relationships in early signaling events of flow-mediated endothelial mechanotransduction. Annu Rev Physiol 59:527-549

Dayton PA et al (1999) Optical and acoustical observations of the effects of ultrasound on contrast agents. IEEE Trans Ultrason Ferroelectr Freq Control 46:220-232

de Jong $\mathrm{N}$ et al (2000) Optical imaging of contrast agent microbubbles in an ultrasound field with a $100-\mathrm{MHz}$ camera. Ultrasound Med Biol 26:487-492 
de Jong N et al (2007) "Compression-only" behavior of phospholipidcoated contrast bubbles. Ultrasound Med Biol 33:653-656

de Jong N et al (2009) Ultrasonic characterization of ultrasound contrast agents. Med Biol Eng Comput 47:861-873

Degroote J, Bathe KJ, Vierendeels J (2009) Performance of a new partitioned procedure versus a monolithic procedure in fluid-structure interaction. Comput Struct 87:793-801

Doinikov AA, Haac JF, Dayton PA (2009) Modeling of nonlinear viscous stress in encapsulating shells of lipid-coated contrast agent microbubbles. Ultrasonics 49:269-275

Fang F, Szleifer I (2001) Kinetics and thermodynamics of protein adsorption: a generalized molecular theoretical approach. Biophys J 80:2568-2589

Ferrara K, Pollard R, Borden M (2007) Ultrasound microbubble contrast agents: fundamentals and application to gene and drug delivery. Annu Rev Biomed Eng 9:415-447

Ferziger JH, Peric M (1995) Computational methods for fluid dynamics. Springer Verlag, Berlin-New York

Fischer M et al (1996) Flow velocity of single lymphatic capillaries in human skin. Am J Physiol Heart C 270:H358-H363

Gorce JM, Arditi M, Schneider M (2000) Influence of bubble size distribution on the echogenicity of ultrasound contrast agents- a study of SonoVue (TM). Invest Radiol 35:661-671

Haidekker MA et al (2002) A novel approach to blood plasma viscosity measurement using fluorescent molecular rotors. Am J Physiol Heart C 282:H1609-H1614

Hernot S, Klibanov AL (2008) Microbubbles in ultrasound-triggered drug and gene delivery. Adv Drug Delivery Rev 60:1153-1166

Hoff L, Sontum PC, Hovem JM (2000) Oscillations of polymeric microbubbles: effect of the encapsulating shell. J Acoust Soc Am 107:2272-2280

Hosseinkhah N et al (2013) Mechanisms of microbubble-vessel interactions and induced stresses: a numerical study. J Acoust Soc Am 134:1875-1885

Hosseinkhah N, Goertz DE, Hynynen K (2015) Microbubbles and blood-brain barrier opening: a numerical study on acoustic emissions and wall stress predictions. IEEE Trans Biomed Eng 62:1293-1304

Hosseinkhah N, Hynynen K (2012) A three-dimensional model of an ultrasound contrast agent gas bubble and its mechanical effects on microvessels. Phys Med Biol 57:785-808

Hsiao CT, Chahine GL (2013) Breakup of finite thickness viscous shell microbubbles by ultrasound: a simplified zero-thickness shell model. J Acoust Soc Am 133:1897-1910

Hynynen K (2008) Ultrasound for drug and gene delivery to the brain. Adv Drug Delivery Rev 60:1209-1217

Hynynen K et al (2005) Local and reversible blood-brain barrier disruption by noninvasive focused ultrasound at frequencies suitable for trans-skull sonications. Neuroimage 24:12-20

Issa RI (1986) Solution of the implicitly discretized fluid-flow equations by operator-splitting. J Comput Phys 62:40-65

Jasak H, Tukovic Z (2007) Automatic mesh motion for the unstructured finite volume method. Trans Famena 30:1-18

Jasak H, Jemcov A, Tukovic Z (2007) OpenFOAM: a C++ library for complex physics simulations. Proc Int Workshop on Coupled Methods in Numerical Dynamics, Dubrovnik, Croatia, pp. 47-66

Klotz AR, Hynynen K (2010) Simulations of the Devin and Zudin modified Rayleigh-Plesset equations to model bubble dynamics in a tube. Electron J Tech Acous 11:1-15

Kuttler U et al (2010) Coupling strategies for biomedical fluid-structure interaction problems. Int J Numer Meth Bio 26:305-321

Li Q et al (2013) Modeling complicated rheological behaviors in encapsulating shells of lipid-coated microbubbles accounting for nonlinear changes of both shell viscosity and elasticity. Phys Med Biol 58:985-998
Liu YY, Miyoshi H, Nakamura M (2006) Encapsulated ultrasound microbubbles: therapeutic application in drug/gene delivery. J Control Release 114:89-99

Lowe GDO (1987) Blood rheology in vitro and in vivo. Baillieres Clin Haematol 1:597-636

Marmottant P et al (2005) A model for large amplitude oscillations of coated bubbles accounting for buckling and rupture. J Acoust Soc Am 118:3499-3505

McDannold N, Vykhodtseva N, Hynynen K (2006) Targeted disruption of the blood-brain barrier with focused ultrasound: association with cavitation activity. Phys Med Biol 51:793-807

McDannold N, Vykhodtseva N, Hynynen K (2008) Blood-brain barrier disruption induced by focused ultrasound and circulating preformed microbubbles appears to be characterized by the mechanical index. Ultrasound Med Biol 34:834-840

McHedlishvili GI, Varazashvili MN (1980) High erythrocyte concentration in blood circulating in the brain. Bull Exp Biol Med 90:1479-1481

Miao H, Gracewski SM, Dalecki D (2008) Ultrasonic excitation of a bubble inside a deformable tube: implications for ultrasonically induced hemorrhage. J Acoust Soc Am 124:2374-2384

Moran CM et al (2000) Quantification of microbubble destruction of three fluorocarbon-filled ultrasonic contrast agents. Ultrasound Med Biol 26:629-639

Nyborg WL (2001) Biological effects of ultrasound: development of safety guidelines. Part II: general review. Ultrasound Med Biol 27:301-333

Patabendige A, Skinner RA, Abbott NJ (2013) Establishment of a simplified in vitro porcine blood-brain barrier model with high transendothelial electrical resistance. Brain Res 1521:1-15

Paul S et al (2010) Material characterization of the encapsulation of an ultrasound contrast microbubble and its subharmonic response: strain-softening interfacial elasticity model. J Acoust Soc Am 127:3846-3857

Plesset MS, Prosperetti A (1977) Bubble dynamics and cavitation. Annu Rev Fluid Mech 9:145-185

Pries AR, Secomb TW, Gaehtgens P (2000) The endothelial surface layer. Pflug Arch Eur J Phy 440:653-666

Putnam FW (1975) The plasma proteins : structure, function, and genetic control. Academic Press, New York

Qin SP, Ferrara KW (2006) Acoustic response of compliable microvessels containing ultrasound contrast agents. Phys Med Biol 51:5065-5088

Quaia E (2007) Microbubble ultrasound contrast agents: an update. Eur Radiol 17:1995-2008

Sarkar K et al (2005) Characterization of ultrasound contrast microbubbles using in vitro experiments and viscous and viscoelastic interface models for encapsulation. J Acoust Soc Am 118:539550

Schlachetzki F et al (2002) Observation on the integrity of the blood-brain barrier after microbubble destruction by diagnostic transcranial color-coded sonography. J Ultrasound Med 21:419_ 429

Secomb TW, Hsu R, Pries AR (2006) Tribology of capillary blood flow. P I Mech Eng J-J Eng 220:767-774

Sheikov N et al (2004) Cellular mechanisms of the blood-brain barrier opening induced by ultrasound in presence of microbubbles. Ultrasound Med Biol 30:979-989

Stride E (2005) Characterization and design of microbubble-based contrast agents suitable for diagnostic imaging. Medical radiology. In: Quaia E (ed) Contrast Media in Ultrasonography. Springer, Berlin Heidelberg, pp 31-42

Stride E, Saffari N (2003a) Microbubble ultrasound contrast agents: a review. P I Mech Eng H 217:429-447 
Stride E, Saffari N (2003b) On the destruction of microbubble ultrasound contrast agents. Ultrasound Med Biol 29:563-573

Stride E, Saffari N (2004) Theoretical and experimental investigation of the behaviour of ultrasound contrast agent particles in whole blood. Ultrasound Med Biol 30:1495-1509

Stucker M et al (2004) Capillary blood cell velocity in periulcerous regions of the lower leg measured by laser Doppler anemometry. Skin Res Technol 10:174-177

$\mathrm{Tu} \mathrm{J}$ et al (2009) Estimating the shell parameters of SonoVue (R) microbubbles using light scattering. J Acoust Soc Am 126:29542962

Tukovic Z, Jasak H (2007) Updated Lagrangian finite volume solver for large deformation dynamic response of elastic body. Trans Famena 31:55-70

Tukovic Z, Jasak H (2012) A moving mesh finite volume interface tracking method for surface tension dominated interfacial fluid flow. Comput Fluids 55:70-84

Unger EC et al (2004) Therapeutic applications of lipid-coated microbubbles. Adv Drug Delivery Rev 56:1291-1314

van der Meer SM et al (2007) Microbubble spectroscopy of ultrasound contrast agents. J Acoust Soc Am 121:648-656

van Wamel A et al (2006) Vibrating microbubbles poking individual cells: drug transfer into cells via sonoporation. J Control Release 112:149-155
Versluis M (2010) Nonlinear behavior of ultrasound contrast agent microbubbles and why shell buckling matters. In: Proceedings of 20th International Congress on Acoustics, Sydney, Australia

Vykhodtseva N, McDannold N, Hynynen K (2008) Progress and problems in the application of focused ultrasound for blood-brain barrier disruption. Ultrasonics 48:279-296

Weller HG et al (1998) A tensorial approach to computational continuum mechanics using object-oriented techniques. Comput Phys 12:620-631

Wiedemair W et al (2012) On ultrasound-induced microbubble oscillation in a capillary blood vessel and its implications for the blood-brain barrier. Phys Med Biol 57:1019-1045

Wiedemair W et al (2014) Modeling the interaction of microbubbles: effects of proximity, confinement, and excitation amplitude. Phys Fluids 26:062106 [1994-present]

Wu JR (2002) Theoretical study on shear stress generated by microstreaming surrounding contrast agents attached to living cells. Ultrasound Med Biol 28:125-129

Ye T, Bull JL (2006) Microbubble expansion in a flexible tube. J Biomech Eng T Asme 128:554-563

Zhang Y et al (2006) Porcine brain microvessel endothelial cells as an in vitro model to predict in vivo blood-brain barrier permeability. Drug Metab Dispos 34:1935-1943 